Original paper

\title{
Toward a pre-clinical irradiator using clinical infrastructure
}

\author{
David Parsons ${ }^{\mathrm{a}}$, Cody Church ${ }^{\mathrm{b}}$, Alasdair Syme ${ }^{\mathrm{c}, *}$ \\ ${ }^{a}$ Department of Radiation Oncology, University of Texas Southwestern Medical Center, 2280 Inwood Road, Dallas, TX 75390-9303, United States \\ ${ }^{\mathrm{b}}$ Department of Physics and Atmospheric Science, Dalhousie University, 5820 University Avenue, Halifax, Nova Scotia B3H 1V7, Canada \\ ${ }^{\mathrm{c}}$ Department of Radiation Oncology and Department of Physics and Atmospheric Science, Dalhousie University, 5820 University Avenue, Halifax, Nova Scotia B3H 1V7, \\ Canada
}

\section{A R T I C L E IN F O}

\section{Keywords:}

Pre-clinical irradiator

Small animal irradiator

Dynamic couch trajectories

Virtual isocentre

\begin{abstract}
A B S T R A C T
Purpose: Pre-clinical irradiation systems use kilovoltage $\mathrm{x}$-ray systems to deliver small fields of radiation in static beam arrangements or arcs. The systems are costly and the radiobiological effectiveness of kilovoltage beams is known to differ from the megavoltage photon beams used clinically. This work used Developer mode on the Varian TrueBeam STx linear accelerator to create a pre-clinical irradiator capable of treating millimeter-sized targets.

Materials and methods: A treatment field defined by a single opposed leaf pair was used to deliver arc-based treatments. Dynamic couch trajectories were used to create a shortened virtual isocentre. Initially, a pre-treatment imaging procedure was used to quantify target misalignment at control points along the arcs and determine appropriate couch positional corrections. This was followed by the treatment arcs in which the positional corrections were implemented. Monte Carlo simulations and radiochromic film were used to calculate and measure dose distributions.

Results: A $1 \mathrm{~mm}$ leaf separation produced the optimal dose distributions. Couch position corrections up to $2.1 \mathrm{~mm}$ were required to maintain a target at virtual isocentre. Application of couch corrections reduced noncoplanar arc treatments dose profile by $1.2 \mathrm{~mm}$ at $30 \%$ of the maximum dose. Treatment of a $1 \mathrm{~mm}$ diameter target would result in falloff distances to the $80 \%, 50 \%$ and $25 \%$ of the $90 \%$ prescription line of $0.3 \mathrm{~mm}, 0.5 \mathrm{~mm}$ and $1.3 \mathrm{~mm}$ from the target edge respectively.

Conclusions: This work has demonstrated that it is possible to deliver highly compact dose distributions using megavoltage photon beams from existing clinical infrastructure.
\end{abstract}

\section{Introduction}

High precision radiotherapy (SRS, SBRT) is a rapidly growing component of the workload of a modern radiation oncology clinic. Ample clinical trial data now demonstrate that the use of high-dose, hypofractionated regimens is capable of efficiently treating small, primary and metastatic disease in the brain [1,2], spine [3], lung [4], liver [5], pancreas [6], kidneys [7] and other sites. High rates of local control are realized while incurring low rates of acute toxicity. The hallmarks of SRS and SBRT treatments typically include high prescription doses (relative to classical $2 \mathrm{~Gy} /$ fraction treatments), rapid dose fall off outside target volumes, small field sizes, reduced PTV margin expansions and exacting standards for machine isocentre dimensions. Currently, SRS and SBRT treatments can be treated with either MLCs or cones.

The ability to treat very small lesions is also a requirement of modern pre-clinical irradiation platforms such as the X-RAD $[8,9]$ family of products (Precision X-ray, North Branford, USA) and the SARRP that was developed at Johns Hopkins University [10-12], among others [13-19]. Pre-clinical studies can be used to investigate the effectiveness of new treatment approaches and may be able to improve the efficiency with which such novel techniques are translated into human patients. In order to mimic human-style radiation therapy, however, small animal irradiation systems must be able to deliver advanced techniques such as non-coplanar arc therapies. The SARRP is capable of producing collimated beams as small as $0.5 \mathrm{~mm}$ in diameter. When treatment is delivered from multiple gantry angles, the irradiated volume can have a FWHM as small as $1.07 \mathrm{~mm}$ [20]. Similarly, the latest X-RAD products are capable of producing beams of $1 \mathrm{~mm}$ diameter. Both systems come with image guidance capabilities, and a tool for platform QA has been developed for the SARRP [21].

In this work we describe methods for the delivery of very compact radiation fields using clinical infrastructure, with beam collimation

\footnotetext{
* Corresponding author.

E-mail addresses: david.parsons@utsouthwestern.edu (D. Parsons), cody.church@dal.ca (C. Church), alasdair.syme@nshealth.ca (A. Syme).
} 

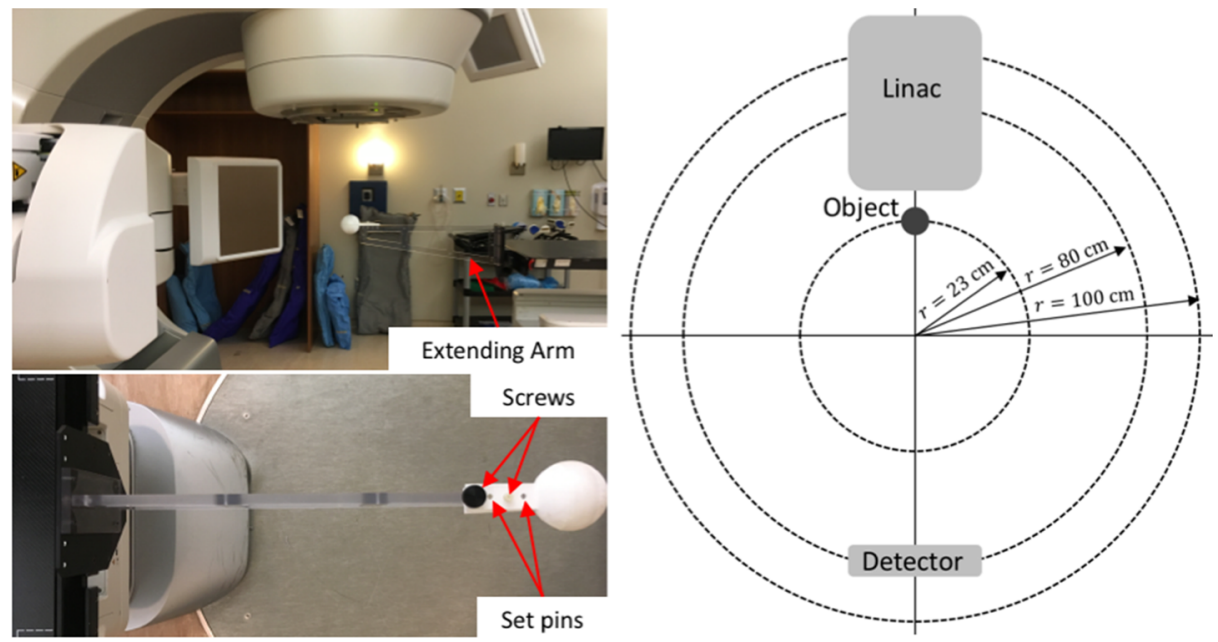

Fig. 1. Illustration and schematic of relative motions for the source, detector and object being irradiated.
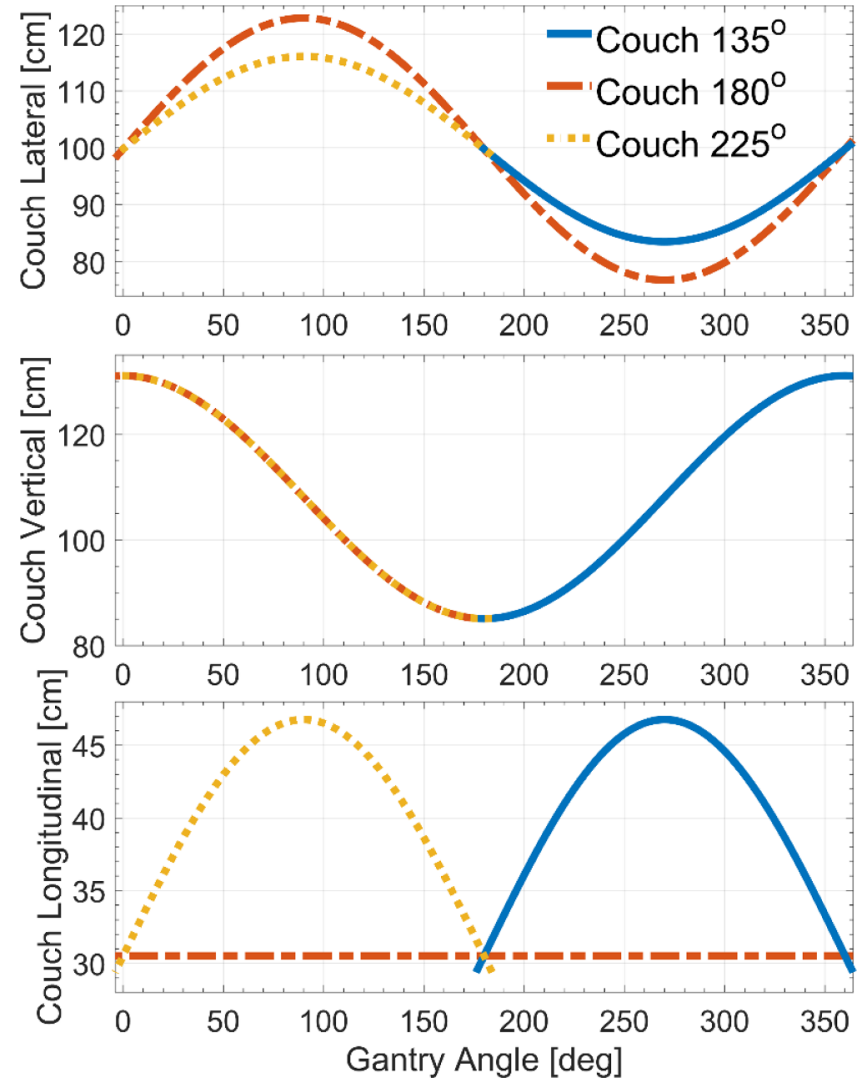

Fig. 2. Couch translations (Varian standard scale, Developer Mode) for couch rotations of $135^{\circ}, 180^{\circ}$ and $225^{\circ}$ and a SAD of $77 \mathrm{~cm}$.

provided by a high-definition MLC. The motivation for this work is twofold. First, pre-clinical irradiators are costly and potentially not available to investigators with interests in in vivo irradiation studies in small animals. In addition, these irradiators make use of kilovoltage x-ray beams with known differences in biological effectiveness relative to megavoltage beams used for clinical treatments [22-24]. For those investigators based in a hospital setting with a radiation oncology department, this work has the potential to offer a tool to perform studies without the requirement for additional significant capital outlay. Concerns related to treating immune-compromised animals in a non-sterile environment and potential exposure of clinical patients to animal dander can be mitigated with good animal handling protocols and room preparation (pre and post treatment). Second, trajectory-based radiation therapy in which the couch and gantry are in simultaneous motion will soon be a clinical reality. One of the potential embodiments of this technology will involve bringing the patient as close as is safely possible to the exit window of the accelerator to realize the benefits of a decreased projected leaf size at virtual isocentre and increased dose rate (i.e. decreased treatment times) primarily driven by inverse square gains. Herein we seek to answer the question: what is the smallest target volume that can be accurately treated by an MLC-defined field?

To answer this question, we created a treatment field that was defined by a single pair of opposed MLC leaves collimated with the smallest allowable jaw-defined field and remaining MLC pairs junctioned under the jaws. We characterized the planar dose distribution as a function of leaf gap, then implemented arc-based deliveries to characterize treatment dose distributions. To maximize the compactness of the distribution, a shortened virtual isocentre was maintained in the treatment aperture by implementing dynamic couch motions. Pretreatment imaging was used to implement couch position perturbations to correct for imperfections in the circular trajectories of the couch/ collimator/gantry. Previous work by Stewart et al. has demonstrated the importance of such couch perturbations to ensure accurate targeting when the subject is placed at the nominal isocentre [25]. To our knowledge, this is the first implementation of a fine couch correction algorithm applied to a virtual isocentre that significantly differs from that of the nominal isocentre.

\section{Materials and methods}

\subsection{Single MLC dosimetry}

The planar dose distribution for a treatment aperture defined by a single opposed leaf pair of the NDS120HD MLC (Varian Medical Systems, Inc., Palo Alto, CA) was measured using gafchromic film (EBT3, Ashland Specialty Ingredients, Bridgewater, NJ) and compared to Monte Carlo simulations using EGSnrc (National Research Council of Canada, Ottawa, Canada). Throughout this manuscript, all field sizes and leaf gaps are specified at nominal isocentre (i.e. $100 \mathrm{~cm} \mathrm{SAD).} \mathrm{The}$ real gaps are reduced proportionally to the ratio of the virtual to nominal isocentre. Leaf gaps ranging from an abutting leaf pair $0.2 \mathrm{~mm}$ separation at isocentre) to a $3.0 \mathrm{~mm}$ opening were studied. All other MLC leaves were positioned behind the $1 \times 1 \mathrm{~cm}^{2}$ jaw-defined field. Distributions were measured using a sheet of gafchromic film positioned between two $30 \times 30 \times 5 \mathrm{~cm}^{3}$ blocks of solid water at a sourceto-surface distance (SSD) of $75 \mathrm{~cm}$. Immediately following irradiation, the film was enclosed in a sealed envelope and twelve hours were 
Table 1

Varian IEC (console display) and Varian standard (Developer Mode) coordinate scales of the relative axes of motion used for virtual isocentre.

\begin{tabular}{lll}
\hline & $\begin{array}{l}\text { Varian IEC } \\
\text { (Console Display) }\end{array}$ & $\begin{array}{l}\text { Varian Standard } \\
\text { (Developer Mode) }\end{array}$ \\
\hline Gantry Rotation & $359^{\circ}-0^{\circ}-1^{\circ}$ & $181^{\circ}-180^{\circ}-179^{\circ}$ \\
Couch Vertical & $999-0-1(\mathrm{~cm})$ & $99-100-101(\mathrm{~cm})$ \\
Couch Longitudinal & $99-100-101(\mathrm{~cm})$ & $99-100-101(\mathrm{~cm})$ \\
Couch Lateral & $999-0-1(\mathrm{~cm})$ & $99-100-101(\mathrm{~cm})$ \\
Couch Rotation & $1^{\circ}-0^{\circ}-359^{\circ}$ & $181^{\circ}-180^{\circ}-179^{\circ}$ \\
\hline
\end{tabular}

allotted for film processing time. Films were scanned with an EPSON EXPRESSION 10,000 XL, at a resolution of $300 \mathrm{dpi}$, with a 48 bit colourdepth. Seven pre-scans were performed to ensure uniform heating of the bulb within the scanner; and films were scanned at the same spatial location on the scanner bed. Absolute dosimetry was calculated with a triple-channel film dosimetry algorithm outlined in Mayer et al. [26].

The measured dose distributions were compared to Monte Carlo simulation of the physical setup. Simulation of treatment head of the TrueBeam STx platform for a $6 \mathrm{MV}$ beam was provided by Varian Medical Systems through 61 phase space files $(\sim 50 \mathrm{~Gb})$ validated against gold beam data down to a field size of $4 \times 4 \mathrm{~cm}^{2}$ [27]. These phase spaces are located $73.3 \mathrm{~cm}$ above isocentre and served as the input for a BEAMnrc [28] model containing the jaws, HDMLC and Mylar exit window. These three components were modeled using exact geometric and material specifications provided by Varian Medical Systems. This was used as an input to DOSXYZnrc [29].

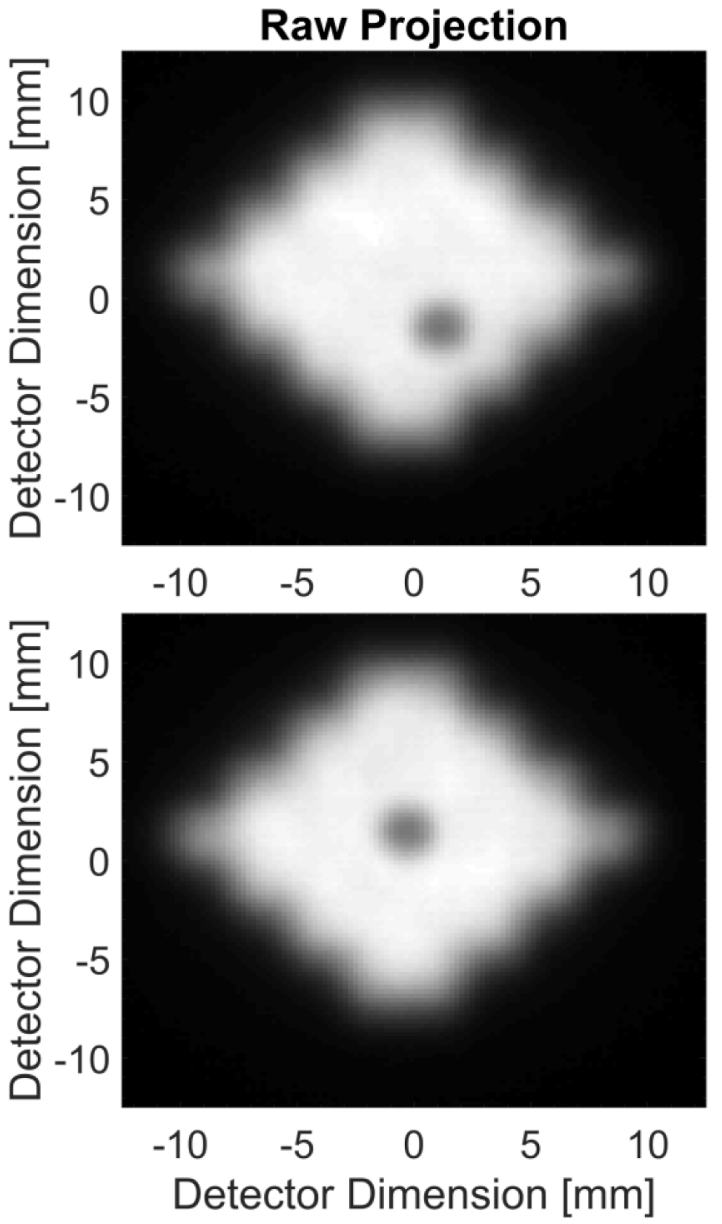

\subsection{Virtual isocentre}

Two $8.0 \mathrm{~cm}$ diameter spheres were 3D printed using polylactic acid (PLA). The spheres were printed in two halves. For one sphere, a divot was machined into both halves to allow a $2.0 \mathrm{~mm}$ ball bearing (BB) to be inserted between them. For the second, a rectangular cavity was removed in each, such that 37 gafchromic sheets of film $\left(40.0 \times 40.0 \times 0.25 \mathrm{~mm}^{3}\right.$ each) could be sandwiched between the two halves. Both spheres included a handle for the purpose of attaching the sphere to the treatment couch using a plastic extension arm (Fig. 1). This permitted either sphere to be reproducibly attached to the arm using two set pins and two screws.

The sphere was initially positioned at the nominal isocentre (i.e. $100 \mathrm{~cm}$ SAD) using the room lasers and $\mathrm{kV}-\mathrm{kV}$ alignment. For subsequent imaging or treatment deliveries at a shortened virtual isocentre, couch positions were calculated as:

$$
\begin{aligned}
\operatorname{Lng}_{\theta, \phi} & =\operatorname{Lng}_{i s o}+r \sin \theta \sin \phi \\
\text { Lat }_{\theta, \phi} & =\text { Lat }_{\text {iso }}+r \sin \theta \cos \phi \\
\operatorname{Vrt}_{\theta, \phi} & =\mathrm{Vrt}_{i s o}+r \cos \theta
\end{aligned}
$$

where $\theta$ is the gantry angle $(-4 \leq \theta \leq 364$, in Varian coordinates), $\mathrm{Lng}_{\text {iso }}, \mathrm{Lat}_{i s o}, \mathrm{Vrt}_{\text {iso }}$ are the initial longitudinal, lateral and vertical couch positions and $\phi$ is the couch angle. The motions are illustrated in Fig. 2. In this work we limited the couch angles to $-45^{\circ}, 0^{\circ}$ or $45^{\circ}$ (corresponding to Varian coordinates of $135^{\circ}, 180^{\circ}$ and $225^{\circ}$, respectively Table 1 contains a comparison of the clinical and Developer Mode coordinate scales). In an ideal mechanical system, these couch

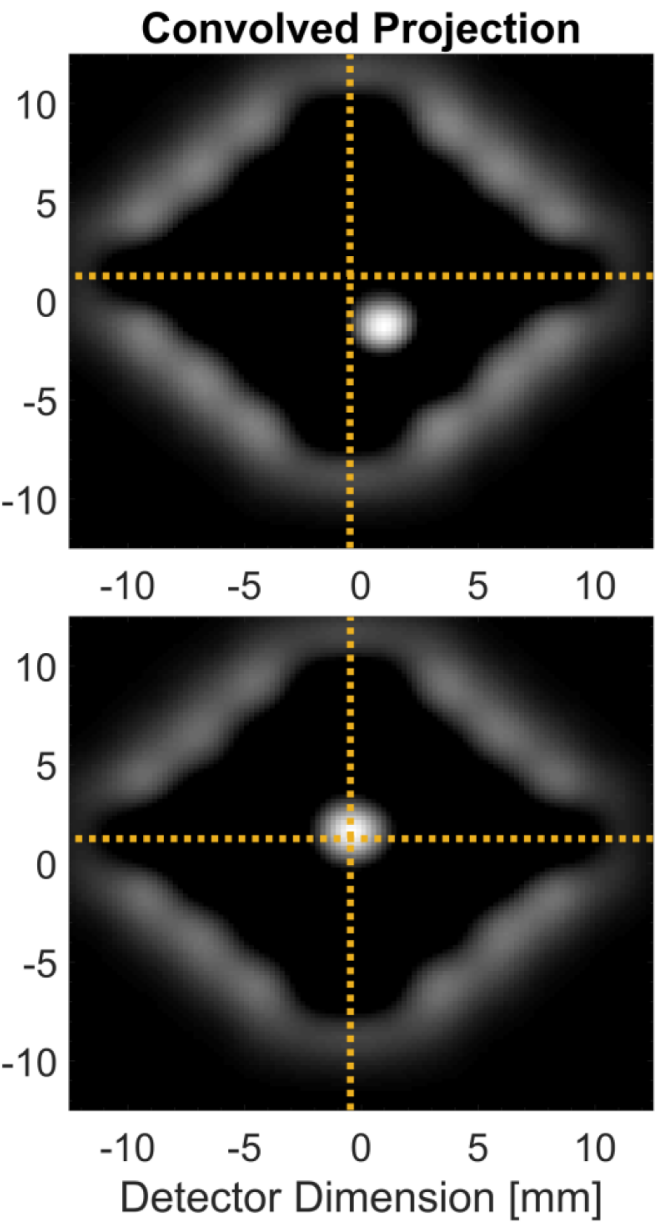

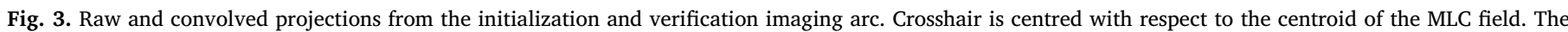

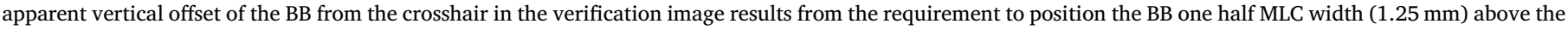
centroid since the treatment field is defined by a single opposed leaf pair. 

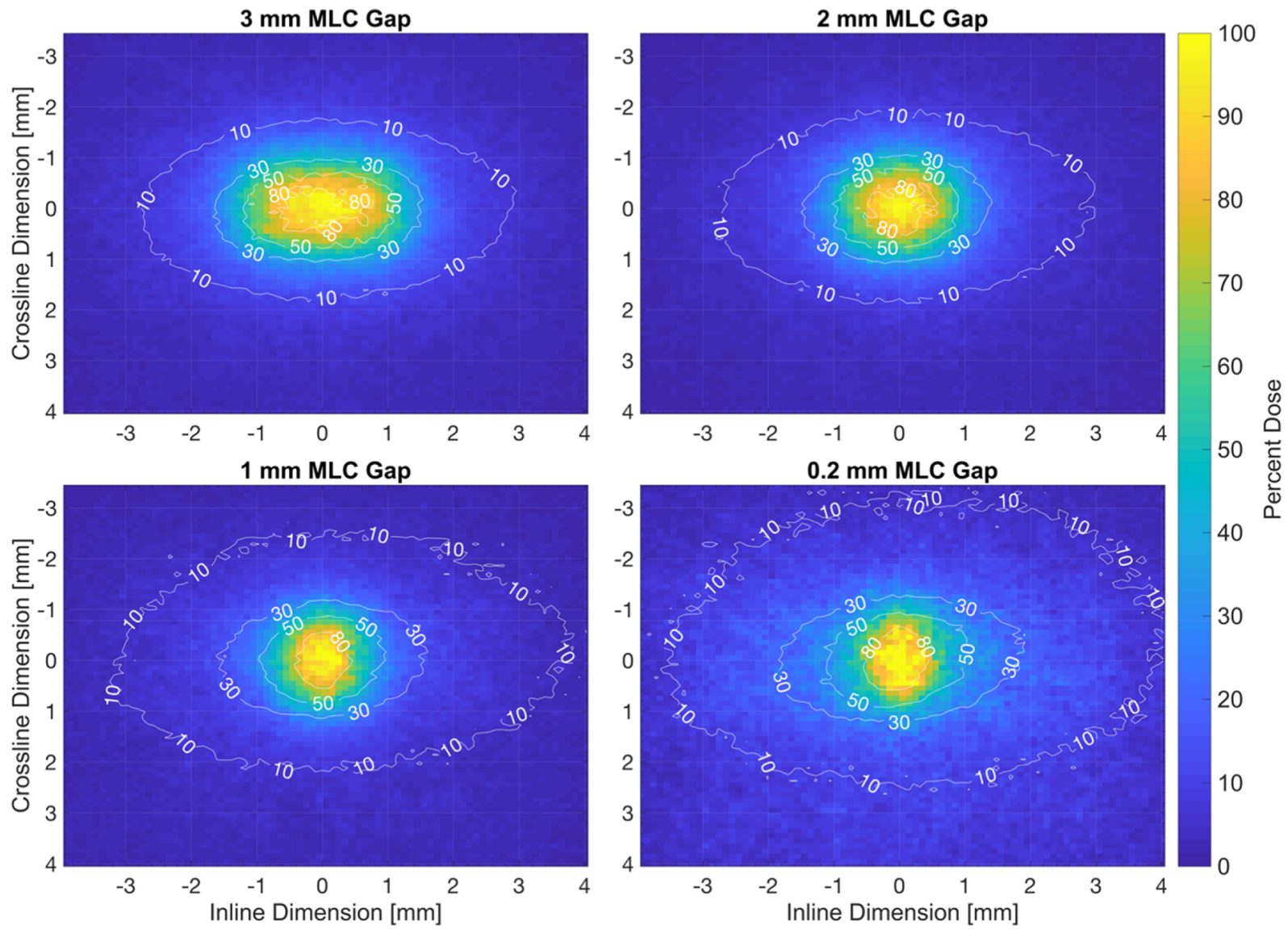

Fig. 4. Monte Carlo (color map) and film (contours) dose distributions for various single MLC leaf separation.

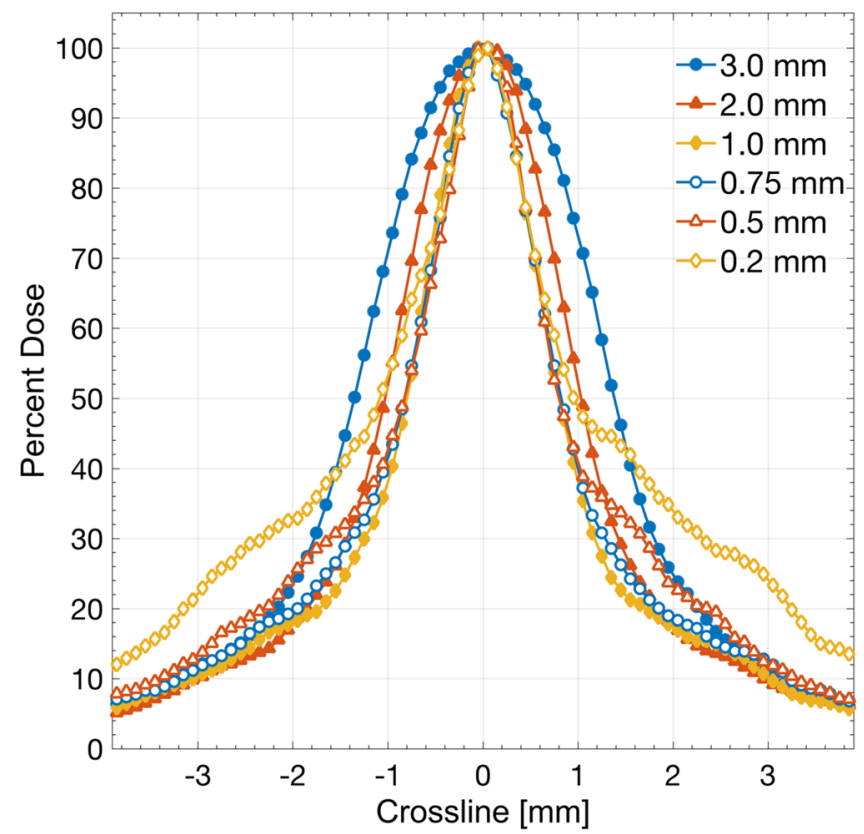

Fig. 5. Monte Carlo simulated dose crossline profiles of various MLC leaf separation ranging from 0.2 to $3.0 \mathrm{~mm}$.

translations would represent the necessary and sufficient corrections to maintain a fixed virtual isocentre. In a real mechanical system like a medical linear accelerator, this is not the case.

To minimize the broadening of the dose distribution caused by nonideal couch, MLC and gantry motions, an initialization imaging arc was acquired in which the $2.5 \mathrm{MV}$ beam was used to image the BB sphere at unit angle gantry increments. The MLC was shaped into a diamond with the centre leaf pair opened to $1.0 \mathrm{~cm}$ each and the three leaves on either side opened to $0.25,0.5$ and $0.75 \mathrm{~cm}$ (Fig. 3). Similar to previous work [30-32], in each projection the BB and field edge were automatically detected using a maximum convolution approach. This involved using a convolution kernel with the central pixels set to negative one and surrounded by a one-pixel border with a positive value such that the total value of the kernel is zero. When convolved with the projection, the result is zero for objects larger than the $\mathrm{BB}$, maximum values for objects with the same size and shape. The resulting convolved projection was then thresholded and binarized. The centroid of the BB and MLC field edge was then calculated and the difference between the two taken

$x=B B_{x}-M L C_{x}$

$y=B B_{y}-M L C_{y}$

were $B B_{x}, B B_{y}, M L C_{x}$ and $M L C_{y}$ are the $\mathrm{x}$-y coordinate of the $\mathrm{BB}$ and MLC field centroid, respectively. The necessary couch translations were then calculated in the non-rotated couch frame as

$\Delta$ Lat $_{\theta, \phi=0}=x \cos \theta$

$\Delta \operatorname{Lng}_{\theta, \phi=0}=y$

$\Delta \operatorname{Vrt}_{\theta, \phi=0}=x \sin \theta$

and then transformed to the rotated couch frame

$\left[\begin{array}{c}\Delta \mathrm{Lat}_{\theta, \phi} \\ \Delta \mathrm{Lng}_{\theta, \phi} \\ \Delta \mathrm{Vrt}_{\theta, \phi}\end{array}\right]=\left[\begin{array}{ccc}\cos \phi & -\sin \phi & 0 \\ \sin \phi & \cos \phi & 0 \\ 0 & 0 & 1\end{array}\right]\left[\begin{array}{c}\Delta \mathrm{Lat}_{\theta, \phi=0} \\ \Delta \mathrm{Lng}_{\theta, \phi=0} \\ \Delta \mathrm{Vrt}_{\theta, \phi=0}\end{array}\right]$

and added or subtracted (due to the Varian coordinate system) from the 

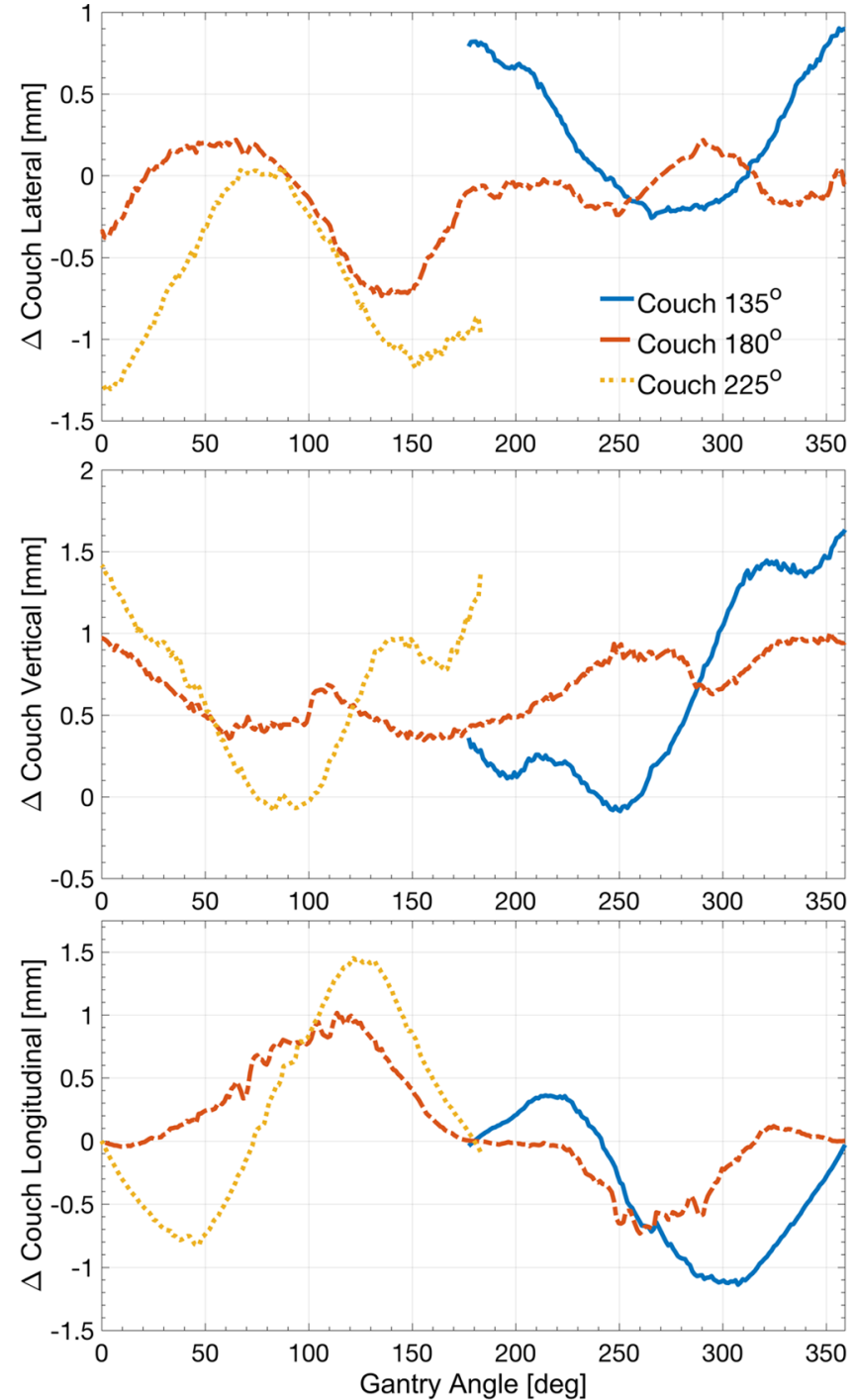

Fig. 6. Magnitude of couch lateral, vertical and longitudinal corrections for couch rotations of $135^{\circ}, 180^{\circ}$ and $225^{\circ}$ (Varian standard scale).

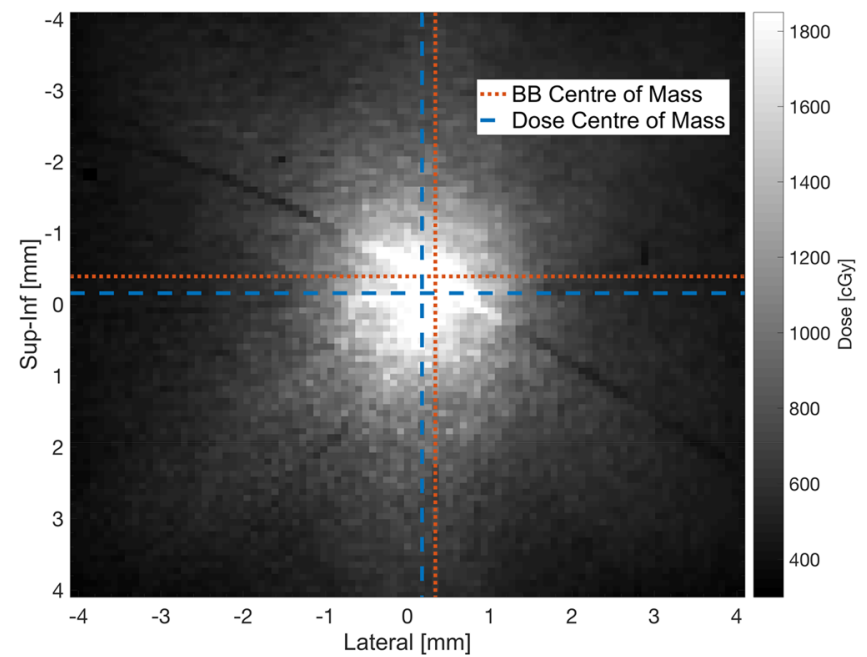

Fig. 7. Co-localization of the therapeutic dose and BB centre of mass, displayed with two crosshairs. original couch location

$\left[\begin{array}{c}\operatorname{Lat}_{\theta, \phi}^{\prime} \\ \operatorname{Lng}_{\theta, \phi}^{\prime} \\ \operatorname{Vrt}_{\theta, \phi}^{\prime}\end{array}\right]=\left[\begin{array}{c}\operatorname{Lat}_{\theta, \phi} \\ \operatorname{Lng}_{\theta, \phi} \\ \operatorname{Vrt}_{\theta, \phi}\end{array}\right]+\left[\begin{array}{c}\Delta \operatorname{Lat}_{\theta, \phi} \\ -\Delta \operatorname{Lng}_{\theta, \phi} \\ -\Delta \operatorname{Vrt}_{\theta, \phi}\end{array}\right]$

A verification imaging arc was then acquired to verify coincidence of the BB and MLC aperture centroid (Fig. 3). With the angle-specific correction applied, a film dose distribution was acquired with a $1.0 \mathrm{~mm}$ single leaf gap using a 6 MVFFF beam, full gantry rotation and a couch angle of zero degrees. Additionally, in a subsequent acquisition two additional half gantry rotations with couch rotations of $\pm 45^{\circ}$ were added in an attempt to reduce the non-target dose of each arc and to increase the compactness of the high dose region.

\subsection{Targeting accuracy}

To examine the targeting accuracy of the technique, a single $40 \times 40 \mathrm{~mm}^{2}$ sheet of film was placed between the two halves of the BB sphere. On one side of the film, a $1 \mathrm{~mm}$ diameter patient positioning $B B$ (SL-10, The Suremark Company, Simi Valley, CA) was attached to the film. This $1 \mathrm{~mm}$ diameter BB filled the divot on one half of the phantom. The initialization imaging arc was then acquired, followed by the 6 MVFFF treatment arc. Twelve hours following exposure, the film with attached BB was first scanned. The BB was then removed and a filmonly scan was acquired. Prior to either scan, four $0.4 \mathrm{~mm}$ pin holes were made in the periphery of the film to permit high-accuracy co-registration of the images. Co-registration, using translations only with mutual information, was used to align the four pin holes in the two scanned images. The centroid of the $\mathrm{BB}$ and high dose was determined using the previously mentioned maximum convolution approach. The targeting accuracy was defined as the distance between the respective centres of mass of the dose distribution and the BB.

\subsection{Treatment planning}

Treatment deliveries with small fields and in small subjects makes planning challenging. This is true for both megavoltage and kilovoltage systems [33]. The use of megavoltage photons to treat targets in a small animal like a mouse could pose challenges related to accurate dosimetric quantification in the buildup region of the treatment field. We have adopted a method in which the target would be maintained at a virtual isocentre while being housed at the center of a holder whose dimensions are large enough to put the target beyond the depth of maximum dose (see figures in Appendix 1). Furthermore, by making the holder spherical, we have reduced the treatment planning problem to a trivial superposition of a kernel. The output factor for the treatment field (i.e. cGy/MU) was determined from calibrated Gafchromic film using standard methods [26]. The EGSnrc model in Section 2.1 was used to simulate the dose distribution for a single $1 \mathrm{~mm}$ single opposed leaf pair on an $8 \mathrm{~cm}$ diameter sphere of water with a voxel dimension of $0.25 \mathrm{~mm}^{3}$. The dose kernel in combination with superposition was used to calculate the dose distribution from the treatment trajectory. For each degree of the treatment delivery, the kernel was rotated to account for gantry, couch and collimator rotation in the sphere coordinate system. Tricubic interpolation was used to map the rotated dose distribution on a Cartesian coordinate system and accumulated over the complete delivered trajectory. The non-coplanar, position-corrected delivery dose distribution (measured with gafchromic film) was compared to a calculation using methods described here. 

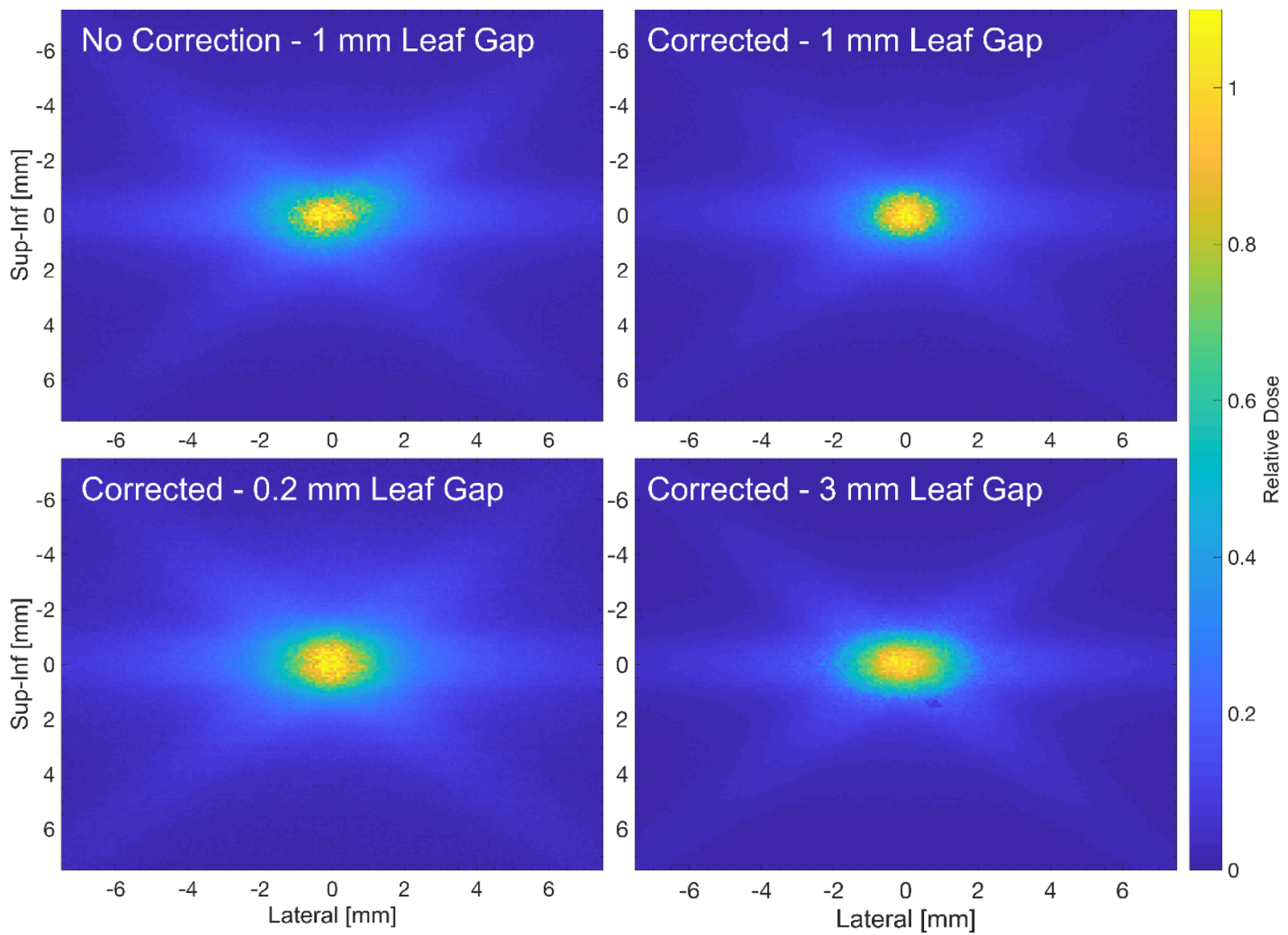

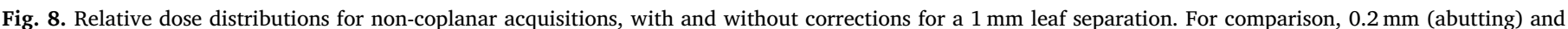
$3 \mathrm{~mm}$ leaf separation is also shown. Coplanar distributions not shown.

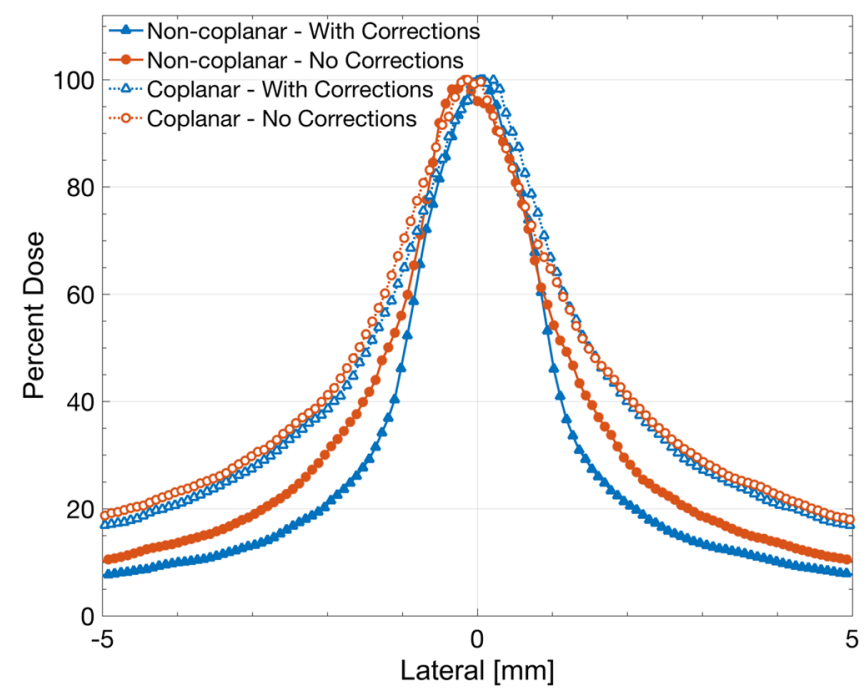

Fig. 9. Crossline profiles for non-coplanar and coplanar dose distributions using a $1 \mathrm{~mm}$ MLC leaf separation, with and without image corrections.

\section{Results and discussion}

\subsection{Single field MLC leaf dosimetry}

Fig. 4 shows Monte Carlo and film dose distributions for four MLC leaf separations at SSD of $75 \mathrm{~cm}$ and a depth of $5 \mathrm{~cm}$. Fig. 5 shows corresponding Monte Carlo crossline profiles (i.e. in leaf motion direction) for MLC leaf separations ranging from 0.2 to $3 \mathrm{~mm}$ (at isocentre). As demonstrated in these two figures a $1 \mathrm{~mm}$ leaf separation provides the most compact dose distribution with lowest peripheral dose. As the MLC gap decreases below $1 \mathrm{~mm}$, the peripheral dose distribution increases with minimal effect on the dose full width at half maximum (FWHM). This is primarily caused by a loss of lateral charged particle equilibrium and occlusion of the primary source [34]. The occlusion effect becomes more dominant as the field size becomes comparable or smaller than the size of the beam spot size (FWHM less than $2 \mathrm{~mm}$ for TrueBeam) and will affect penumbra fall-off. As Varian does not provide the detailed specifications of the head of the TrueBeam accelerator (to permit independent investigation of spot size effects), no further small field dosimetric validation was performed on the linac simulation model. Above $1 \mathrm{~mm}$, the FWHM increases with gap width and converges with the $1 \mathrm{~mm}$ peripheral dose.

\subsection{Dosimetric effect of image corrections}

Fig. 6 shows the calculated corrections to couch motion as a function of gantry angle for couch angles of $135^{\circ}, 180^{\circ}$ and $225^{\circ}$. The maximum magnitude of couch motion were $2.1 \mathrm{~mm}, 1.1 \mathrm{~mm}$ and $1.9 \mathrm{~mm}$ for couch angles of $135^{\circ}, 180^{\circ}$ and $225^{\circ}$, respectively, while the maximum in any one direction for all three arcs was $1.6 \mathrm{~mm}$.

Fig. 7 shows the results of the targeting accuracy study in which a sheet of gafchromic film was co-localized with a $1 \mathrm{~mm} \mathrm{BB}$. The distance between the centres of mass of the dose and the $\mathrm{BB}$ was $0.3 \mathrm{~mm}$.

The use of megavoltage photons to deliver small fields of radiation will never produce comparable beam penumbra characteristics to an orthovoltage irradiation system, largely due to the relatively high energy of the Compton scattered electrons and their significant lateral range. However, when using modern, non-coplanar arc therapies, a 

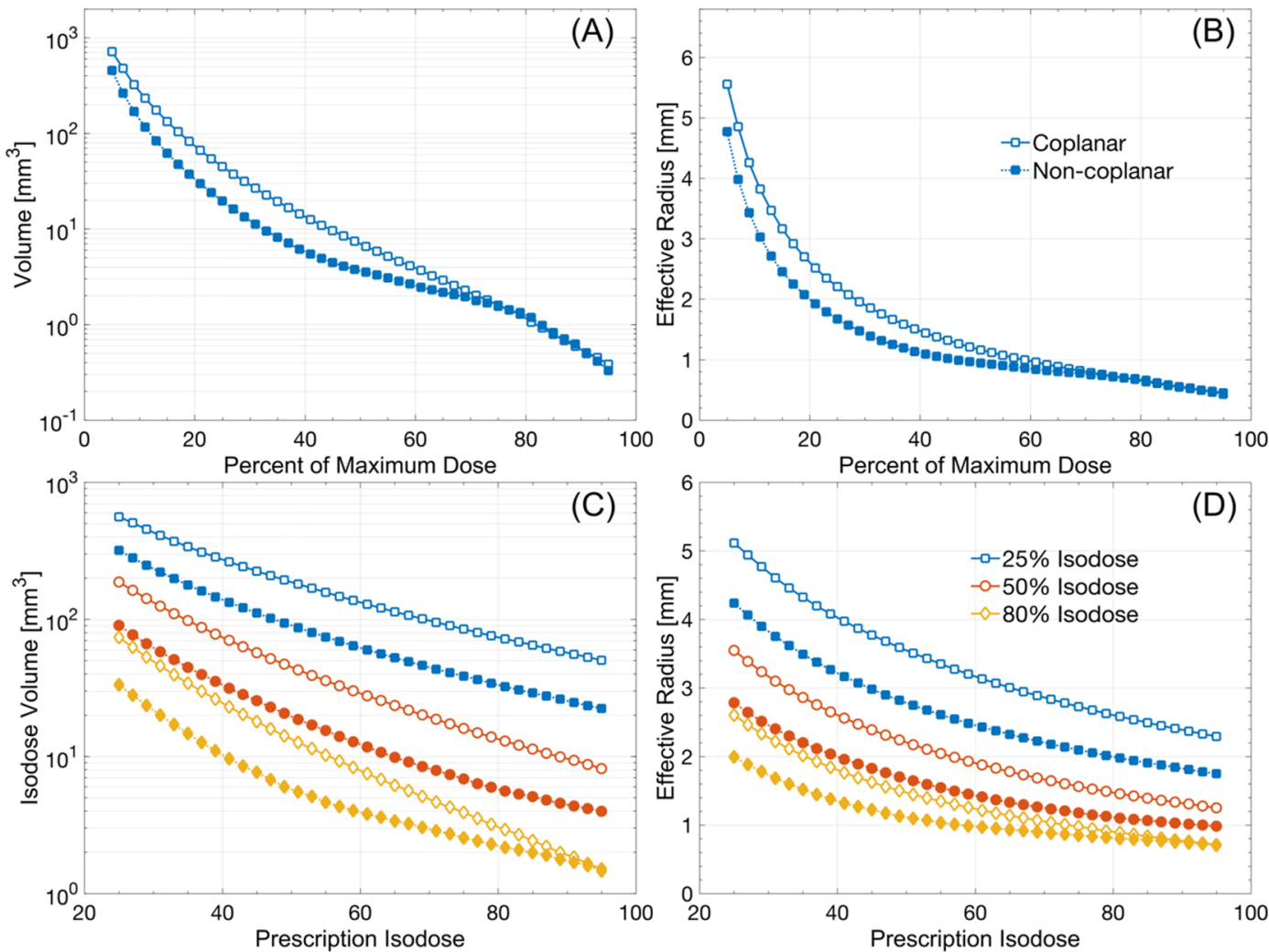

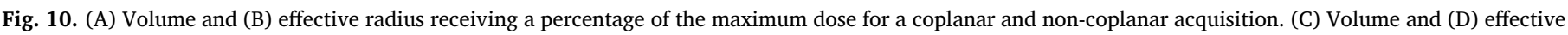

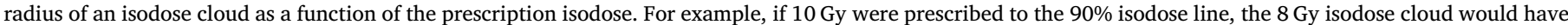

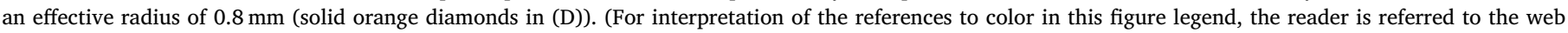
version of this article.)

megavoltage photon beam is capable of producing a highly compact dose distribution. In this work, we have shown that a radiation field shaped by a single MLC leaf pair can effectively treat millimeter sized targets with rapid dose fall off characteristics when dynamic couch motions are implemented to reduce the distance between the target and the MLC. These data can be compared with the previously reported FWHM value of $1.07 \mathrm{~mm}$ for the SARRP platform [20]. Furthermore, targeting accuracy at the level of $0.3 \mathrm{~mm}$ can be achieved indicating that the system has excellent potential for applications in pre-clinical studies. Herein we have not studied the role of kilovoltage imaging in this system, though the presence of an on board imager would certainly facilitate such a feature.

Fig. 8 shows relative dose distributions for non-coplanar acquisitions for an isocentre of $77 \mathrm{~cm}$ with and without corrections for the $1 \mathrm{~mm}$ leaf gap, and with corrections for the $0.2 \mathrm{~mm}$ and $3 \mathrm{~mm}$ gaps. The effect of corrections with respect to dose compactness is readily apparent for the $1 \mathrm{~mm}$ gap films, as is the enhanced relative intensity of the low dose wash for the $0.2 \mathrm{~mm}$ gap. Fig. 9 shows corresponding crossline dose profiles for coplanar and non-coplanar acquisitions using a $1 \mathrm{~mm}$ MLC leaf separation both with and without corrections. As demonstrated, the application of image corrections results in a more compact distribution. This is more significant for the non-coplanar acquisition, in which doses below approximately $75 \%$ of the maximum dose diverge from the corrected profile and add $1.2 \mathrm{~mm}$ to the field width at the $30 \%$ dose level.

Fig. 10 presents differences in the 3-dimensional dose volumes between a coplanar and non-coplanar acquisition for a $1 \mathrm{~mm}$ leaf gap. For this analysis, the full gafchromic film stack was considered. The maximum dose in the distribution was taken as an average of the 75 highest dose voxel elements (a $5 \times 5 \times 3$ voxel array centred on the global maximum dose). A data point in Fig. 10a then corresponds to the total volume of space encompassed by the isodose level ( $\mathrm{x}$-axis) expressed as a percentage of the maximum dose. The same data are shown in Fig. 9b, but the spatial metric is presented as an effective radius for the dose cloud (this helps facilitate comparison with published results for other small animal irradiator systems). As shown in Fig. 10a-b, above $75 \%$ of the maximum dose, the difference between a coplanar and non-coplanar acquisitions is minimal, however below this level the two diverge, and the dose fall off is significantly more rapid in the non-coplanar delivery. Fig. 9c-d are intended to illustrate the dose fall off characteristics of the system. For example, if one intended to treat a target volume with a radius of $0.5 \mathrm{~mm}$ with the non-coplanar geometry, the target would be covered by the $90 \%$ isodose line (Fig. 10b). Fig. 10d can be used to gain some understanding of the rate at which the dose falls off outside of the target volume. In the hypothetical example of a $0.5 \mathrm{~mm}$ radius target, the radial distance (from the centre of the dose distribution) at which the dose would fall to $80 \%, 50 \%$ and $25 \%$ of the $90 \%$ prescription line would then be $0.8 \mathrm{~mm}, 1 \mathrm{~mm}$ and $1.8 \mathrm{~mm}$ respectively (Fig. 10d). If the fall off outside the target is quantified by subtracting the target radius $(0.5 \mathrm{~mm})$, those values become $0.3 \mathrm{~mm}$, $0.5 \mathrm{~mm}$ and $1.3 \mathrm{~mm}$ from the target edge respectively.

\subsection{Volumetric dosimetry with MLC leaf separation}

In Fig. 11, the volume receiving percentage of the maximum dose and effective radius (assuming a spherical distribution) is shown. The data for the $1 \mathrm{~mm}$ gap are the same as those shown in Fig. 10a-b (noncoplanar) and are included here for comparison with the dose volume data for the $0.2 \mathrm{~mm}$ gap and the $3 \mathrm{~mm}$ gap. For doses larger than approximately $50 \%$ of the maximum dose, the $3 \mathrm{~mm}$ leaf separation produces isodose volumes that are larger than the $0.2 \mathrm{~mm}$ leaf gap. However, below $45 \%$, the two diverge and the enhanced low dose wash of the $0.2 \mathrm{~mm}$ gap produces larger isodose volumes. Below $20 \%$ of the maximum dose the $3 \mathrm{~mm}$ and $1 \mathrm{~mm}$ gaps produce isodose volumes that 


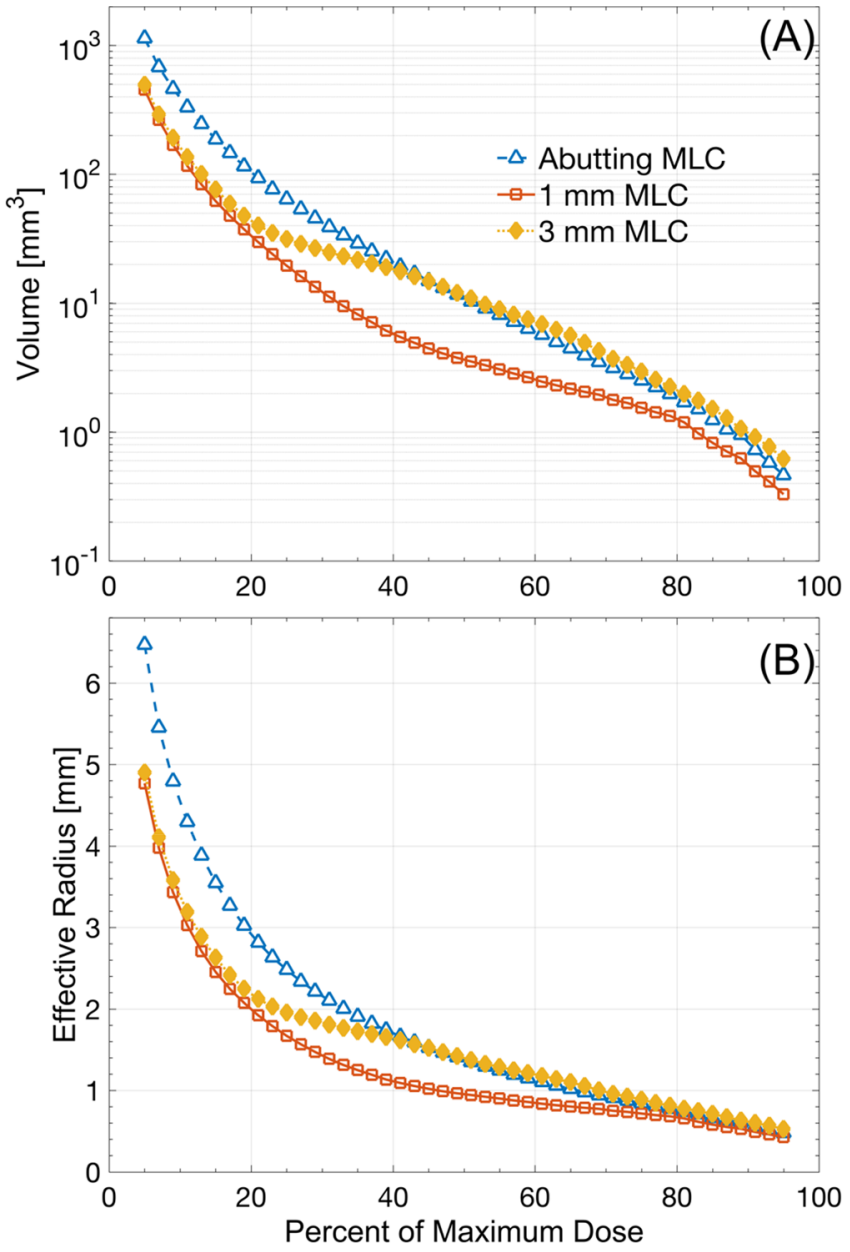

Fig. 11. (A) Volume and (B) effective radius receiving a percentage of the maximum dose for a $0.2 \mathrm{~mm}$ (abutting), $1 \mathrm{~mm}$ and $3 \mathrm{~mm}$ MLC leaf separation.

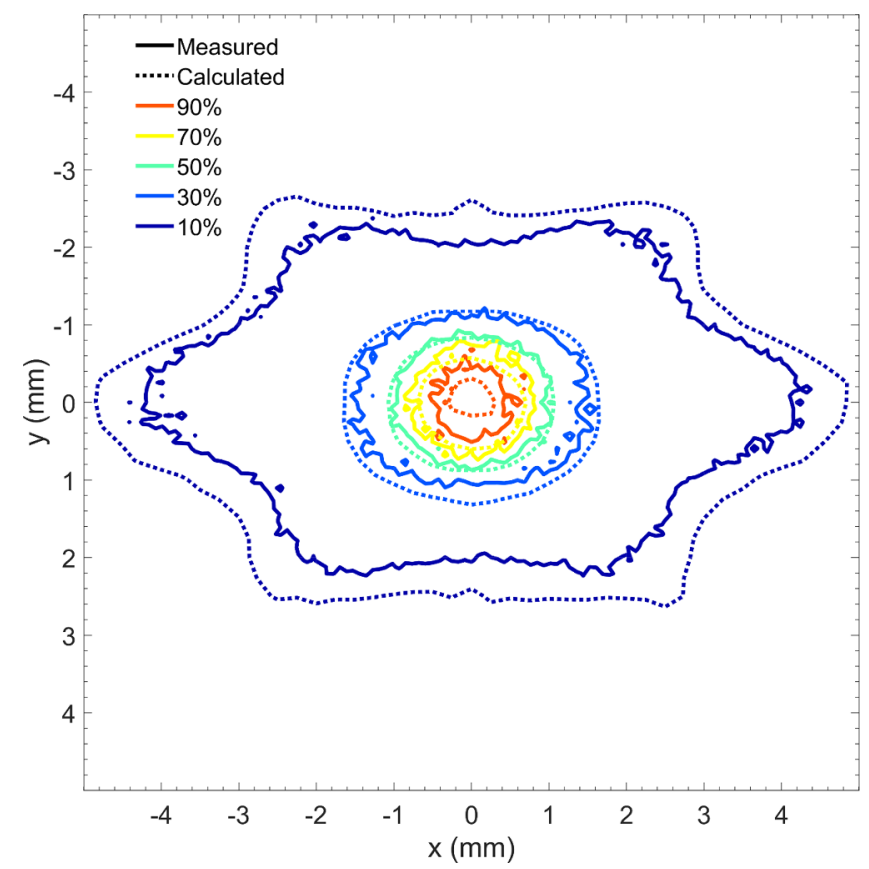

Fig. 12. Calculated and corrected film percent dose distributions for a noncoplanar delivery.
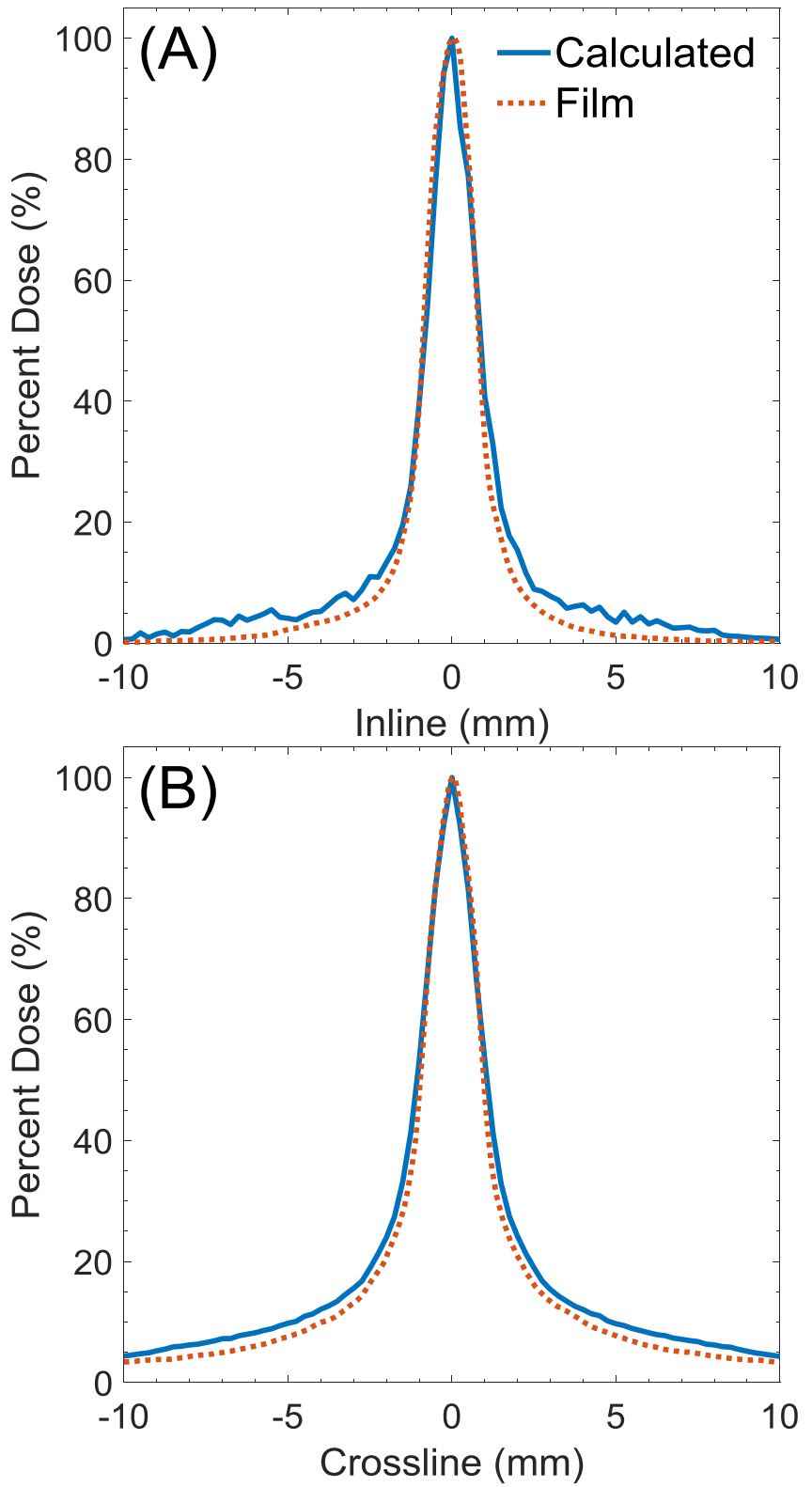

Fig. 13. Calculated and corrected film inline (a) and crossline (b) dose profiles for the non-coplanar delivery.

begin to converge, which is consistent with the convergence of the beam profiles observed in Fig. 5.

\subsection{Treatment planning}

Fig. 12 shows the comparison between calculated (i.e. Monte Carlo kernel plus superposition) and measured film dose distributions for the non-coplanar delivery. The measured distribution conforms to the calculated distribution to within the voxel spacing used. The exception to this is in the low dose fall-off (less than $20 \%$ of the maximum), in which the film reports a lower dose than the Monte Carlo predicts. This is further demonstrated in Fig. 13. For doses down to approximately $30 \%$ of the maximum value, the distance to agreement between the measured and calculated doses is less than $0.3 \mathrm{~mm}$. Below $30 \%$ of the maximum, the distance to agreement between the profiles can be as large as $0.6 \mathrm{~mm}$, with the calculated profile being larger than the measured profile. For the purposes of pre-clinical studies, this small discrepancy in the low dose region is likely of little concern.

The output of the system was determined using calibrated film 
measurements. At a depth of $4 \mathrm{~cm}$, the $1 \mathrm{~mm}$ leaf gap produced an output of $0.401 \pm(0.037) \mathrm{cGy} / \mathrm{MU}$. In the example shown in Fig. 12, the $4000 \mathrm{MU}$ used to deliver the treatment resulted in a maximum dose (at virtual isocentre) of $1604 \pm$ (151) cGy. Clearly the output of the system will depend strongly on the exact separation of the leaf pair (i.e. it would likely be insufficient to simply specify a nominal leaf gap of $1 \mathrm{~mm}$ ) and each system would require a specific calibration of the output as well as an appropriate quality assurance program to ensure dosimetric accuracy at the time of treatment delivery. We have evaluated the reproducibility of the output of the system for the $1 \mathrm{~mm}$ treatment field by retracting and re-asserting the MLCs and measuring the dose with gafchromic film. In multiple series of 10 exposures (acquired over multiple weeks), we found reproducibility of approximately $1 \%$ in each series (data not shown).

For the spherical phantom used, the kernel and superposition method was sufficient for accurate dose calculation. Treatment planning with reduced or no buildup material would increase the complexity and in future work, a full Monte Carlo calculation method will be explored. Reducing the buildup material would potentially permit the conformal targeting of even smaller tumours due to a reduction in scatter, however, even with the current setup, we have demonstrated the accurate targeting of millimeter-sized targets with rapid dose fall off. Although mouse or rat bones are unlikely to significantly perturb a dose distribution, the presence of lung tissue in the treatment field during an arc will be studied in future work.

\section{Conclusions}

In this work, we have demonstrated that a clinical linear accelerator is capable of treating millimeter-sized targets with rapid dose fall-off, sub-millimeter targeting accuracy and biological equivalence relative to clinical therapeutic techniques. For non-coplanar treatments, the twostep approach utilized herein results in reduction of the dose profile by $1.2 \mathrm{~mm}$ at the $30 \%$ isodose level compared to an uncorrected acquisition. Additionally, for a treatment of a $1 \mathrm{~mm}$ diameter target prescribed to the $90 \%$ isodose line would produce falloff distances to the $80 \%$, $50 \%$ and $25 \%$ of the $90 \%$ prescription line of $0.3 \mathrm{~mm}, 0.5 \mathrm{~mm}$ and $1.3 \mathrm{~mm}$ from the target edge, respectively. These improvements are realized without substantial changes to the clinical system; as it is already permitted by the linac platform, albeit through Developer Mode.

\section{Financial disclosure}

No disclosures.

\section{Acknowledgement}

The authors would like to thank Ian Porter for fabrication of apparatus essential in completing this work.

\section{Appendix 1}

\section{Example irradiator setup}

The benefits of a relatively large ( $8 \mathrm{~cm}$ diameter) spherical sample holder include significant simplification of monitor unit and dose distribution calculations, as well as the flexibility to hold the sample in a number of configurations to facilitate irradiation of targets in different parts of the animal anatomy. By using 3D-printing techniques, the internal surfaces of the two halves of the sphere can conform to the body of the animal to ensure reproducible positioning In the following images, the top half of the sphere has been removed for visualization purposes.

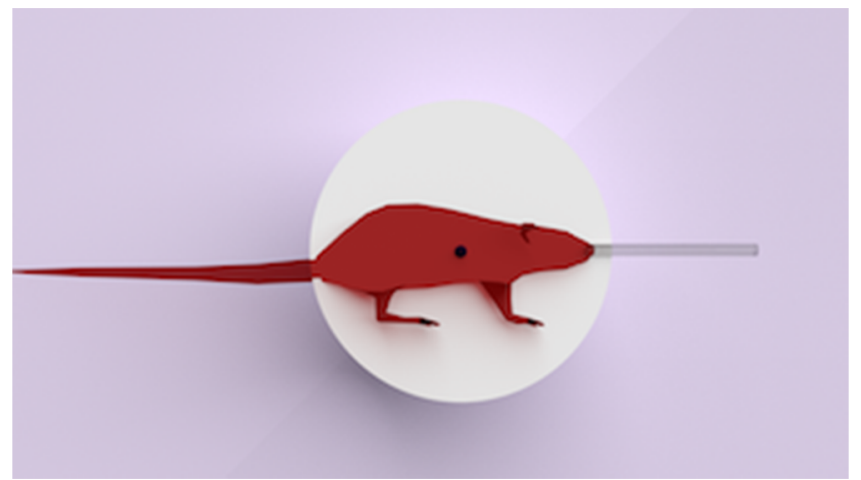

Fig. A1. Positioning of a mouse within the spherical holder for a central target.

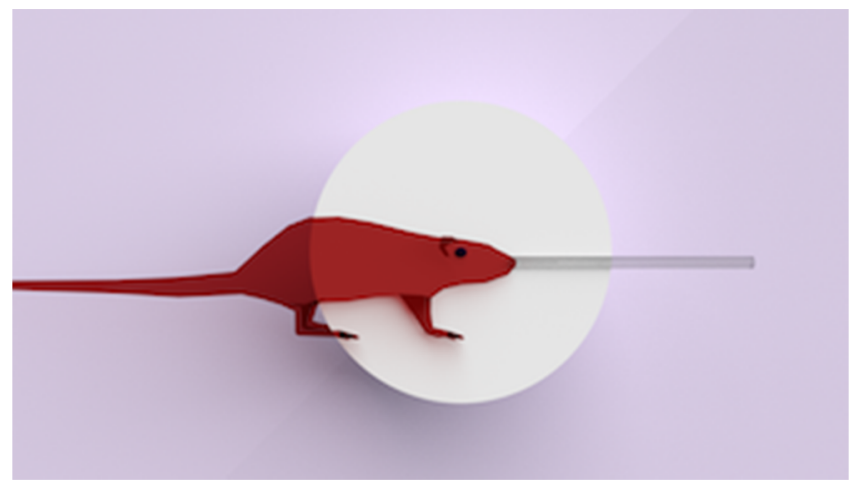

Fig. A2. Positioning of a mouse within the spherical holder for a cranial target. 


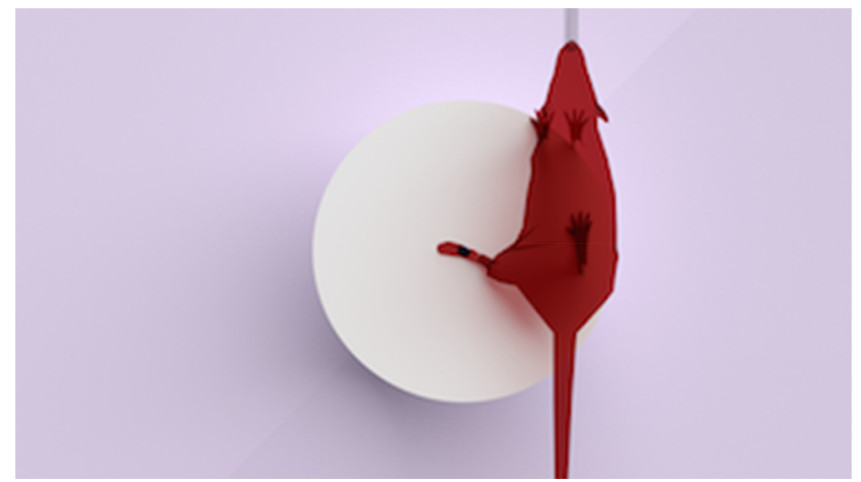

Fig. A3. Positioning of a mouse within the spherical holder for a target on the extremity.

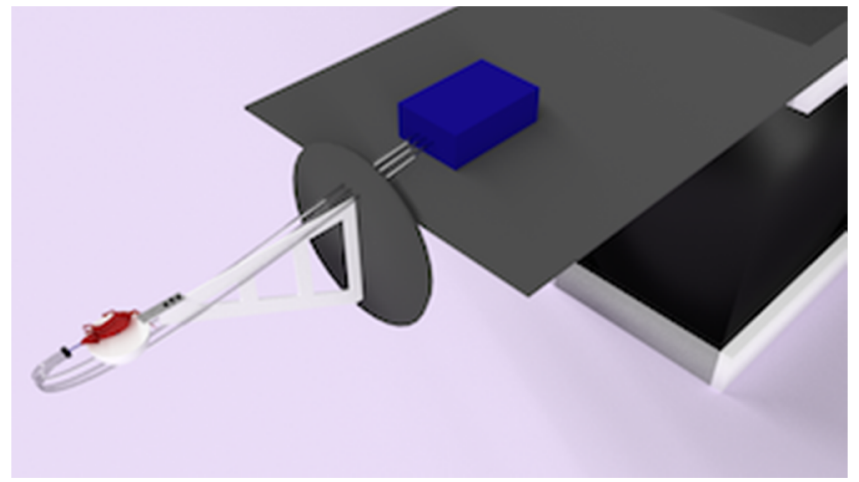

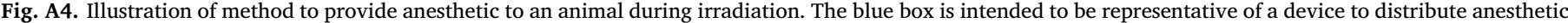
(send and scavenge), send and receive feedback pertaining to relevant biometric data (e.g. heart rate, temperature) during treatment.

\section{References}

[1] Andrews DW, Scott CB, Sperduto PW, Flanders AE, Gaspar LE, Schell MC, et al Whole brain radiation therapy with or without stereotactic radiosurgery boost for patients with one to three brain metastases: phase III results of the RTOG 9508 randomised trial. Lancet 2004:363:1665-72.

[2] Kondziolka D, Patel A, Lunsford LD, Kassam A, Flickinger JC. Stereotactic radiosurgery plus whole brain radiotherapy versus radiotherapy alone for patients with multiple brain metastases. Int J Radiat Oncol Biol Phys 1999;45:427-34.

[3] Calcerrada Diaz-Santos N, Blasco Amaro JA, Cardiel GA, Andradas Aragones E. The safety and efficacy of robotic image-guided radiosurgery system treatment for intraand extracranial lesions: a systematic review of the literature. Radiother Onco $2008 ; 89: 245-53$

[4] Onishi H, Shirato H, Nagata Y, Hiraoka M, Fujino M, Gomi K, et al. Stereotactic body radiotherapy (SBRT) for operable stage I non-small-cell lung cancer: can SBRT be comparable to surgery? Int J Radiat Oncol Biol Phys 2011;81:1352-8.

[5] Schefter TE, Kavanagh BD, Timmerman RD, Cardenes HR, Baron A, Gaspar LE. A phase I trial of stereotactic body radiation therapy (SBRT) for liver metastases. Int J Radiat Oncol Biol Phys 2005;62:1371-8.

[6] Chang DT, Schellenberg D, Shen J, Kim J, Goodman KA, Fisher GA, et al. Stereotactic radiotherapy for unresectable adenocarcinoma of the pancreas. Cancer 2009; 115:665-72.

[7] Svedman C, Karlsson K, Rutkowska E, Sandstrom P, Blomgren H, Lax I, et al. Stereotactic body radiotherapy of primary and metastatic renal lesions for patients with only one functioning kidney. Acta Oncol 2008;47:1578-83.

[8] Clarkson R, Lindsay PE, Ansell S, Wilson G, Jelveh S, Hill RP, et al. Characterization of image quality and image-guidance performance of a preclinical microirradiator. Med Phys 2011;38:845-56.

[9] Stewart JM, Lindsay PE, Jaffray DA. Two-dimensional inverse planning and delivery with a preclinical image guided microirradiator. Med Phys 2013:40:101709.

[10] Deng H, Kennedy CW, Armour E, Tryggestad E, Ford E, McNutt T, et al. The smallanimal radiation research platform (SARRP): dosimetry of a focused lens system. Phys Med Biol 2007;52:2729-40.

[11] Matinfar M, Ford E, Iordachita I, Wong J, Kazanzides P. Image-guided small animal radiation research platform: calibration of treatment beam alignment. Phys Med Biol 2009;54:891-905.

[12] Wong J, Armour E, Kazanzides P, Iordachita I, Tryggestad E, Deng H, et al. Highresolution, small animal radiation research platform with $\mathrm{x}$-ray tomographic guidance capabilities. Int J Radiat Oncol Biol Phys 2008;71:1591-9.
[13] Graves EE, Zhou H, Chatterjee R, Keall PJ, Gambhir SS, Contag CH, et al. Design and evaluation of a variable aperture collimator for conformal radiotherapy of small animals using a microCT scanner. Med Phys 2007;34:4359-67.

[14] Kiehl EL, Stojadinovic S, Malinowski KT, Limbrick D, Jost SC, Garbow JR, et al. Feasibility of small animal cranial irradiation with the microRT system. Med Phys 2008;35:4735-43.

[15] Pidikiti R, Stojadinovic S, Speiser M, Song KH, Hager F, Saha D, et al. Dosimetric characterization of an image-guided stereotactic small animal irradiator. Phys Med Biol 2011;56:2585-99.

[16] Rodriguez M, Zhou H, Keall P, Graves E. Commissioning of a novel microCT/RT system for small animal conformal radiotherapy. Phys Med Biol 2009;54:3727-40.

[17] Song KH, Pidikiti R, Stojadinovic S, Speiser M, Seliounine S, Saha D, et al. An X-ray image guidance system for small animal stereotactic irradiation. Phys Med Biol 2010;55:7345-62.

[18] Stojadinovic S, Low DA, Hope AJ, Vicic M, Deasy JO, Cui J, et al. MicroRT-small animal conformal irradiator. Med Phys 2007;34:4706-16.

[19] Zhou H, Rodriguez M, van den Haak F, Nelson G, Jogani R, Xu J, et al. Development of a micro-computed tomography-based image-guided conformal radiotherapy system for small animals. Int J Radiat Oncol Biol Phys 2010;78:297-305.

[20] Verhaegen F, Granton P, Tryggestad E. Small animal radiotherapy research platforms. Phys Med Biol 2011;56:R55.

[21] Jermoumi M, Korideck H, Bhagwat M, Zygmanski P, Makrigiogos GM, Berbeco RI, et al. Comprehensive quality assurance phantom for the small animal radiation research platform (SARRP). Phys Med 2015;31:529-35.

[22] Amols HI, Lagueux B, Cagna D. Radiobiological effectiveness (RBE) of megavoltage X-ray and electron beams in radiotherapy. Radiat Res 1986;105:58-67.

[23] Hill MA. The variation in biological effectiveness of X-rays and gamma rays with energy. Radiat Prot Dosimetry 2004;112:471-81.

[24] Ibbott GS. Radiation dosimetry: electron beams with energies between 1 and 50 MeV (ICRU report No. 35). Med Phys 1985;12:813-.

[25] Stewart JM, Ansell S, Lindsay PE, Jaffray DA. Online virtual isocenter based radiation field targeting for high performance small animal microirradiation. Phys Med Biol 2015;60:9031.

[26] Mayer RR, Ma F, Chen Y, Miller RI, Belard A, McDonough J, et al. Enhanced dosimetry procedures and assessment for EBT2 radiochromic film. Med Phys 2012;39:2147-55.

[27] Constantin M, Perl J, LoSasso T, Salop A, Whittum D, Narula A, et al. Modeling the truebeam linac using a CAD to Geant4 geometry implementation: dose and IAEAcompliant phase space calculations. Med Phys 2011;38:4018-24.

[28] Rogers DW, Faddegon BA, Ding GX, Ma CM, We J, Mackie TR. BEAM: a Monte Carlo 
code to simulate radiotherapy treatment units. Med Phys 1995;22:503-24.

[29] Walters B, Kawrakow I, Rogers DWO. DOSXYZnrc users manual. Ottawa, Canada: National Research Council of Canada; 2018.

[30] Parsons D, Stevens MT, Robar JL. Current modulated volume-of-interest imaging for kilovoltage intrafaction monitoring of the prostate. Med Phys 2017;44:1479-93.

[31] Stevens MTR, Parsons DD, Robar JL. Continuous monitoring of prostate position using stereoscopic and monoscopic kV image guidance. Med Phys 2016;43:2558-68.

[32] Stevens MTR, Parsons DD, Robar JL. Patient specific methods for room-mounted X- ray imagers for monoscopic/stereoscopic prostate motion monitoring. J Appl Clin Med Phys 2017:18:40-50.

[33] Noblet C, Chiavassa S, Paris F, Supiot S, Lisbona A, Delpon G. Underestimation of dose delivery in preclinical irradiation due to scattering conditions. Phys Med 2014;30:63-8.

[34] Palmans H, Andreo P, Huq MS, Seuntjens J, Christaki K, Alfonso R, et al. Dosimetry of small static fields used in external beam radiotherapy: an IAEA-AAPM international Code of Practice for reference and relative dose determination Technical Reports Series No 483 Vienna, Austria: International Atomic Energy Agency; 2018. 